\title{
Avoidance of Early Post-Operative Intraperitoneal Chemotherapy (EPIC) Following Peritonectomy with Heated Intraperitoneal Chemotherapy (HIPEC) Significantly Reduces Cost and Hospital Stay
}

\author{
Rachel E. Kirby, Jing Zhao, Terence Chua, Winston Liauw and David L. Morris* \\ Hepatobiliary and Surgical Oncology Unit, UNSW Department of Surgery, St. George Hospital, Sydney, Australia
}

\begin{abstract}
Aims: Peritoneal carcinomatosis is treated with Cytoreductive surgery (CRS) and Heated Intraperitoneal Chemotherapy (HIPEC) with or without Early Postoperative Intraperitoneal Chemotherapy (EPIC) depending on the pathology involved. Since 2010 heated intraperitoneal oxaliplatin has been utilised in our institution. Our aim was to determine if there was a significant cost difference between patients receiving oxaliplatin and those requiring five additional days of EPIC.

Methods: We retrospectively analysed 30 patients from our database. 15 patients underwent cytoreductive surgery, heated intraperitoneal oxaliplatin $\left(350 \mathrm{mg} / \mathrm{m}^{2}\right)$ and 5 -fluorouracil (FU) intravenously administered intraoperatively. We compared those patients with 15 patients who underwent CRS, heated intraperitoneal mitomycin $\mathrm{C}\left(10-25 \mathrm{mg} / \mathrm{m}^{2}\right)$ as well as EPIC with 5-FU $\left(600-800 \mathrm{mg} / \mathrm{m}^{2}\right)$ on postoperative days 1 to 5 . Patients were matched for age, gender, pathology and peritoneal carcinomatosis index (PCI).

Results: There was no significant difference between radiology $(\mathrm{p}=0.6)$ and transfusion costs $(\mathrm{p}=0.4)$. The chemotherapy costs in the oxaliplatin group were significantly higher $(\mathrm{p}=0.001)$ however overall bed costs including ward, HDU and

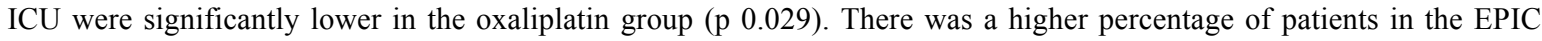
group that had postoperative ileus ( $\mathrm{p} 0.4$ ), rate of infection ( $\mathrm{p} 0.1$ ), fistula formation ( $\mathrm{p} 0.03$ ), return to theatre ( $\mathrm{p} 0.2)$ and collection formation ( $\mathrm{p}$ 0.07) compared to the oxaliplatin group. The overall cost was significantly lower in the oxaliplatin group (p 0.038). There was no significant survival benefit between both groups ( $\mathrm{p} 0.3)$.

Conclusion: In colorectal cancer the cost and complications of EPIC following HIPEC are unjustified. We now use oxaliplatin HIPEC as our standard regimen in colorectal cancer and appendiceal adenocarcinoma.
\end{abstract}

Keywords: Cost analysis, cytoreductive surgery, peritonetomy.

\section{INTRODUCTION}

Cytoreductive surgery (CRS) and hyperthermic intraperitoneal chemotherapy (HIPEC) is now a common treatment for pseudomyxoma peritonei (PMP), malignant mesothelioma, peritoneal carcinomatosis (PC) from colorectal cancer (CRC) and appendiceal carcinoma, currently supported by survival, morbidity and mortality data [1-5].

HIPEC with mitomycin C followed by early postoperative intraperitoneal chemotherapy (EPIC) with 5-fluorouracil (FU) was the first regimen widely utilised based on Sugarbaker's work [6].

More recent data predominantly from France has shown that oxaliplatin has achieved good results and may have some advantage because of the avoidance of EPIC [7, 8].

In a study by Elias et al., CRS and HIPEC with oxaliplatin, a median survival of 63 months was achieved and $51 \% 5$ year survival [9].

*Address correspondence to this author at the University of New South Wales, Department of Surgery, St. George Hospital, Kogarah, NSW 2217, Sydney, Australia; Tel: (02) 911320720; Fax: 029113997;

E-mail: david.morris@unsw.edu.au
Patients with PC with a peritoneal carcinomatosis index (PCI) score less than 19 have a 5 year survival of $29 \%$ and if the score is greater than 19 have a five year survival of $7 \%$ following CRS and HIPEC [10].

Since 2010 we have changed our protocol for colorectal cancer peritonectomy and omitted the five days of EPIC with 5-FU which was our previous standard with MMC HIPEC.

There is currently no randomised controlled trial of these two regimens.

There is limited data on the cost of cytoreductive surgery and there are no comparable studies of the cost of oxaliplatin versus mitomycin $\mathrm{C}$ intraperitoneal treatment. Our aim was to determine if there was a significant cost difference between patients receiving oxaliplatin and those requiring five additional days of early postoperative intraperitoneal chemotherapy (EPIC).

\section{METHODS}

We retrospectively analysed 30 patients from a prospective database of 677 patients who have undergone peritonectomy at our institution including 190 cases of colorectal cancer. 
15 consecutive patients underwent cytoreductive surgery, heated intraperitoneal oxaliplatin $\left(350 \mathrm{mg} / \mathrm{m}^{2}\right)$ at $41.5^{\circ}$ for 30 minutes at a flow rate of $1 \mathrm{~L} / \mathrm{min}$ and 5-fluorouracil (FU) intravenously $(600-800 \mathrm{mg})$ administered intraoperatively from the beginning of 2010 to our most recent.

We compared those patients with 15 matched patients who underwent $\mathrm{CRS}$, heated intraperitoneal mitomycin $\mathrm{C}$ $\left(10-25 \mathrm{mg} / \mathrm{m}^{2}\right)$ at $41.5^{\circ}$ for 90 minutes at flow rate of $1 \mathrm{~L} / \mathrm{min}$ as well as EPIC with 5 -FU $\left(600-800 \mathrm{mg} / \mathrm{m}^{2}\right)$ on postoperative days 1 to 5 .

Patients were randomly selected from our database but matched for age, gender and peritoneal carcinomatosis index (PCI). A cost analysis was performed comparing groups with regard to cost of chemotherapy, length of stay in HDU/ICU and on the ward, radiology and transfusions requirements. We also analysed our most recent survival statistics for colorectal cancer as well as mesothelioma, Peritoneal Mucinous Carcinomatosis (PMCA) and Disseminated Peritoneal Adenomucinosis (DPAM). Groups were analysed using paired two tailed t-test where appropriate. Results were deemed statistically significant with a $\mathrm{p}$ value $<0.05$.

\section{RESULTS}

There was no significant difference between the two groups with regard to gender ( 10 female, 5 male) age $(p=$ $0.6)$ and PCI ( $\mathrm{p}=0.8)$ as illustrated in Table 1.

The mean operative time was 9 hours in the oxaliplatin group and $7.7 \mathrm{hrs}$ in the HIPEC/EPIC group (0.1).

There was no significant difference between radiology $(\mathrm{p}=0.6)$ and transfusion costs $(\mathrm{p}=0.4)$. The chemotherapy costs in the oxaliplatin group were significantly higher $(p=0.001)$ however overall bed costs including ward, HDU and ICU were significantly lower in the oxaliplatin group $(\mathrm{p}=0.029)$. Details are outlined in Table 2 and Graph 1.

Postoperative complications are outlined in Table $\mathbf{3}$. There was a higher percentage of patients in the HIPEC/EPIC group that had a postoperative ileus ( $p 0.4$ ),

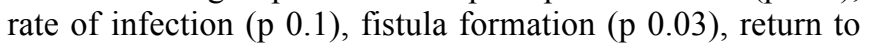
theatre ( $p 0.2$ ) and collection formation (p 0.07) compared to the oxaliplatin group. We had no reported anastomotic leaks in our reported thirty patients.

The HIPEC (mitomycin C) and EPIC group had a higher percentage of patients with grade 4 morbidity (three cases versus seven). There was no significant difference between both groups with regard to survival. In the oxaliplatin group the mean mortality time from surgery was 13 months compared to 22 months (p 0.3) in the HIPEC/EPIC group. The St. George Hospital's survival data is outlined in Table 4. The five year survival for colorectal cancer in our institute is twenty percent with a mean PCI of 19.

\section{DISCUSSION}

Cytoreductive surgery and HIPEC provides an important chance of long term survival in patients with peritoneal spread of colorectal cancer $[1,11]$.

Peritoneal spread of colorectal cancer was associated with death in all patients and a limited and often symptomatic survival until the advent of peritonectomy. The incidence of peritoneal spread of colorectal cancer at the time of presentation has been well described by a number of groups.

Table 1. Comparison of outcome following surgery and heated intraperitoneal chemotherapy (HIPEC) with oxaliplatin versus surgery and HIPEC (mitomycin C) and early postoperative intraperitoneal chemotherapy (EPIC).

\begin{tabular}{|c|c|c|c|c|c|c|}
\hline & HIPEC Oxaliplatin & Standard Deviation & HIPEC Mitomycin/EPIC & Standard Deviation & p Value & Standard Deviation \\
\hline \hline Gender (Male:Female) & $4: 11$ & & $4: 11$ & & \\
\hline Age (mean) & 55 & 11 & 55.5 & 11 & 0.6 & 11 \\
\hline PCI (mean) & 13 & 9.7 & 12.9 & 6.4 & 0.95 \\
\hline LOS (days) & & & & 6.8 & 0.3 & \\
\hline ICU & 3 & 2.3 & 4.5 & 3 & 0.006 & 5 \\
\hline HDU & 2.4 & 2 & 5 & 11 & 0.02 & 3 \\
\hline Ward & 12 & 8.7 & 22 & 12 & 0.0005 & 11 \\
\hline Total & 17 & 10 & 34 & & 13 \\
\hline
\end{tabular}

Table 2. Comparison of cost in patients following surgery and heated intraperitoneal chemotherapy (HIPEC) with oxaliplatin versus surgery and HIPEC (mitomycin C) and early postoperative intraperitoneal chemotherapy (EPIC).

\begin{tabular}{|c|c|c|c|c|}
\hline Mean Cost (Aust Dollars) & HIPEC Oxaliplatin & Standard Deviation & HIPEC Mitomycin/EPIC & Standard Deviation \\
\hline Ward Costs & 6162 & 6058 & 11144 & 15059 \\
\hline Radiology & 496 & 503 & 595 & 486 \\
\hline Blood Products & 2218 & 1875 & 3217 & 0.029 \\
\hline Chemotherapy & 552 & 52 & 501 & 0.4 \\
\hline Overall Cost & 3591 & 4970 & 6040 & 0.001 \\
\hline
\end{tabular}




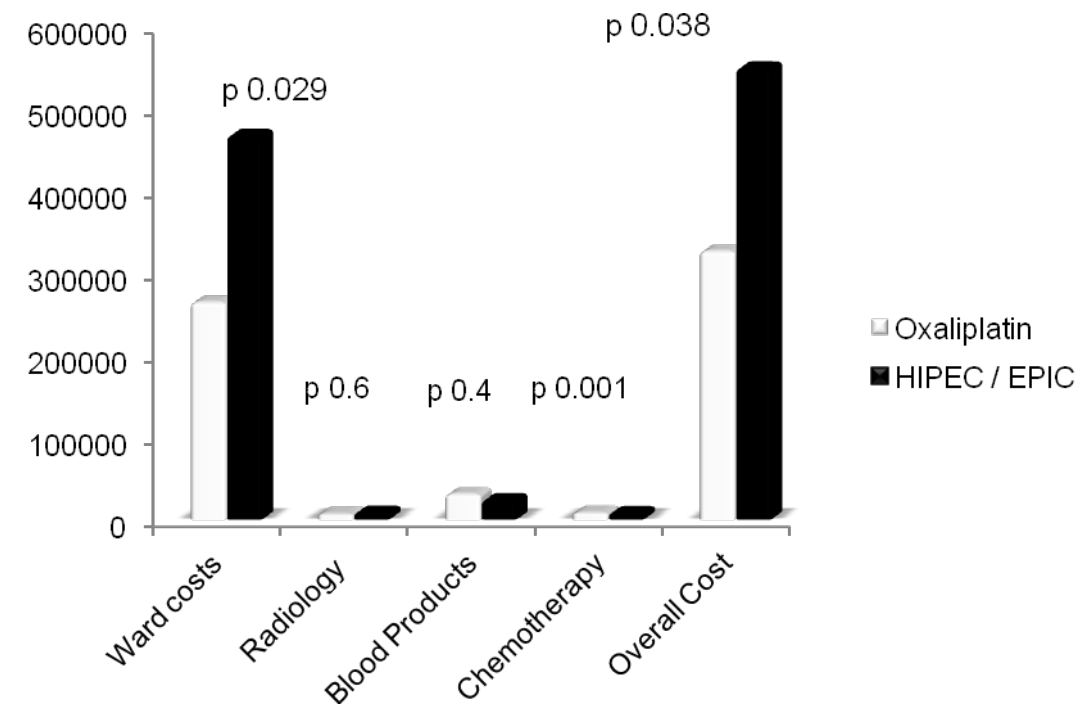

Graph 1. Cost Analysis: heated intraperitoneal chemotherapy (HIPEC) with oxaliplatin versus HIPEC with mitomycin C and early postoperative intraperitoneal chemotherapy (EPIC). (Australian Dollars).

Peritonectomy has however been associated with significant morbidity and mortality associated with a learning curve outlined by several institutions that have identified improved results but do not identify the factors which have created this improvement [12-15].

Table 3. Comparison of complications in patients following surgery and HIPEC (oxaliplatin) versus surgery and HIPEC ( mitomycin C) and EPIC.

\begin{tabular}{|c|c|c|c|}
\hline \multirow{2}{*}{ Complications } & HIPEC Oxaliplatin & HIPEC/EPIC & \multirow{2}{*}{ p Value } \\
\cline { 2 - 3 } & Number (\%) & Number (\%) & \\
\hline \hline Infection & $4(27)$ & $8(53)$ & 0.1 \\
\hline Fistula & 0 & $4(27)$ & 0.03 \\
\hline Ileus & $3(20)$ & $5(33)$ & 0.4 \\
\hline Collection & $4(27)$ & $9(60)$ & 0.07 \\
\hline Return to Theatre & $2(13)$ & $5(33)$ & 0.2 \\
\hline
\end{tabular}

$214(8 \%)$ patients were diagnosed with synchronous peritoneal carcinomatosis in a study that included 2756 patients with colorectal cancer [16].

Recently, two population-based studies reported the incidence of synchronous peritoneal carcinomatosis in the Netherlands (4.8\%) and in Sweden (4.3\%) $[17,18]$.
A further proportion of patients will develop peritoneal carcinomatosis in their follow up.

Following curative resection for colon cancer metachronous peritoneal carcinomatosis is reported in $4 \%$ to $12 \%$ of patients in $2 \%$ to $19 \%$ of patients following curative resection for rectal cancer [19].

Following primary curative resection and undergoing repeat procedures for colorectal cancer, $21 \%$ to $44 \%$ of patients are diagnosed with peritoneal tumour deposits [20].

The evidence base for peritonectomy/HIPEC in colorectal cancer is now very strong in appropriately selected patients with a randomized trial demonstrating a survival benefit of cytoreductive surgery and intraperitoneal chemotherapy over systemic chemotherapy alone $[5,9,10$, 21-23]. Elias et al. have demonstrated a cure rate (16\%) after complete CRS of colorectal peritoneal carcinomatosis, followed by intraperitoneal chemotherapy in selected patients is close to that obtained after resection of colorectal liver metastases [1].

It is also true however that selection of patients is critically important both in terms of excluding patients with other untreatable metastatic disease or a PCI of greater than 20.

The five year survival for colorectal cancer in our institute is twenty percent with a mean PCI of 19. Currently in our institution patients with colorectal cancer are considered for peritonectomy with a PCI less than 15 with no

Table 4. St. George Hospital results for peritonectomy for Peritoneal Mucinous Carcinomatosis (PMCA), Disseminated Peritoneal Adenomucinosis (DPAM), mesothelioma and colorectal cancer.

\begin{tabular}{|c|c|c|c|c|}
\hline Pathology & Procedure Number & PCI (Mean) & Mean Survival (Months) & 5 Year Survival (\%) \\
\hline \hline PMCA & 118 & 20 & 65 & 133 \\
\hline DPAM & 218 & 23 & 63 & 77 \\
\hline Mesothelioma & 55 & 19 & 39 & 53 \\
\hline Colorectal Cancer & 190 & 10 & 20 \\
\hline
\end{tabular}


liver metastases or less than 10 with three or less resectable liver metastases.

The two peritonectomy regimens for intraperitoneal chemotherapy following complete cytoreduction is one of the original regimens of Sugarbaker, HIPEC with mitomycin $\mathrm{C}$ and EPIC with 5-Flurouracil and the more recent French regimens of HIEC with oxaliplatin.

Mitomycin $\mathrm{C}$ is the most frequently used intraperitoneal chemotherapy agent for peritoneal carcinomatosis of colorectal and appendiceal origin [24].

Mitomycin $\mathrm{C}$ is not without complications such as renal toxicity, haemolytic uremic syndrome, impaired wound healing that can contribute to bowel perforation or anastomotic leak or fistula formation. It has been suggested that 5-Fluorouracil used in conjunction with mitomycin $\mathrm{C}$ for five consecutive days of EPIC may be important for long term survival particularly in disseminated peritoneal adenomucinosis (DPAM), however EPIC has its own consequences and is thought to be associated with prolonged ileus and anastomotic failure but has not been previously been compared as in our study [25, 26].

There are side effects which are associated with intraperitoneal oxaliplatin as with all chemotherapeutic agents. The use of 5\% dextrose in the chemoperfusion can lead to solute and electrolyte shifts across the mesothelial barrier as well as elevated blood glucose levels. This can be combatted with careful intraoperative intravenous supplementation and continuous insulin infusion.

Our study clearly shows that cost is significantly reduced (p 0.038 ) by the avoidance of EPIC, this is not a trivial cost in our study as the ward costs were halved. The reason for the cost saving is because of shorter hospital stay and we found that the fistula rate was significantly lower ( $p$ 0.03) in the HIPEC alone group. This again is not of trivial importance in the post op recovery in a peritonectomy patient. We presume that the higher fistula rate in the EPIC group is due to the effects of 5-FU on anastomotic healing [27].

We conclude that in CRC the cost and complications of EPIC following HIPEC are unjustified. Whether there is any good reason to continue EPIC 5-FU in DPAM should also be addressed. We now use oxaliplatin HIPEC as our standard regimen in colorectal cancer and appendiceal adenocarcinoma.

\section{CONFLICT OF INTEREST}

The authors confirm that this article content has no conflict of interest.

\section{ACKNOWLEDGEMENTS}

Pharmacy, Finance and Radiology departments in St. George Hospital.

Peritonectomy team in St. George Hospital Sydney, Australia.

\section{REFERENCES}

[1] Goéré D, Malka D, Elias D, et al. Is there a possibility of a cure in patients with colorectal peritoneal carcinomatosis amenable to complete cytoreductive surgery and intraperitoneal chemotherapy. Ann Surg 2013; 257(6): 1065-71.
[2] Munkholm-Larsen S, Cao CQ, Yan TD. Malignant peritoneal mesothelioma. World J Gastrointest Surg 2009; 1(1): 38-48.

[3] Chua TC, Moran BJ, Morris DL, et al. Early- and long-term outcome data of patients with pseudomyxoma peritonei from appendiceal origin treated by a strategy of cytoreductive surgery and hyperthermic intraperitoneal chemotherapy. J Clin Oncol 2012; 30(20): 2449-56.

[4] Yan TD, Welch L, Black D, Sugarbaker PH. A systematic review on the efficacy of cytoreductive surgery combined with perioperative intraperitoneal chemotherapy for diffuse malignancy peritoneal mesothelioma. Ann Oncol 2007; 18(5): 827-34.

[5] Cao C, Yan TD, Black D, Morris DL. A systematic review and meta-analysis of cytoreductive surgery with perioperative intraperitoneal chemotherapy for peritoneal carcinomatosis of colorectal origin. Ann Surg Oncol 2009; 16(8): 2152-65.

[6] Sugarbaker PH. Peritonectomy procedures. Ann Surg 1995; 221(1): 29-42.

[7] McConnell YJ, Mack LA, Francis WP, Ho T, Temple WJ. HIPEC+EPIC versus HIPEC-alone: Differences in major complications following cytoreduction surgery for peritoneal malignancy. J Surg Oncol 2012; 107(6): 591-6.

[8] Elias D, Benizri E, Di Pietrantonio D, Menegon P, Malka D, Raynard B. Comparison of two kinds of intraperitoneal chemotherapy following complete cytoreductive surgery of colorectal peritoneal carcinomatosis. Ann Surg Oncol 2007; 14(2): 509-14.

[9] Elias D, Lefevre JH, Bonastre J, et al. Complete cytoreductive surgery plus intraperitoneal chemohyperthermia with oxaliplatin for peritoneal carcinomatosis of colorectal origin. J Clin Oncol 2009; 27(5): 681-5.

[10] Glehen O, Kwiatkowski F, Sugarbaker PH, et al. Cytoreductive surgery combined with perioperative intraperitoneal chemotherapy for the management of peritoneal carcinomatosis from colorectal: a multi- institutional study. J Clin Oncol 2004; 22: 3284-92.

[11] Yan TD, Black D, Savady R, et al. Systematic review on the efficacy of cytoreductive surgery combined with perioperative intraperitoneal chemotherapy for peritoneal carcinomatosis from colorectal carcinoma. J Clin Oncol 2006; 24: 4011-9.

[12] Kusamura S, Baratti D, Deraco M. Multidimensional analysis of the learning curve for cytoreductive surgery and hyperthermic intraperitoneal chemotherapy in peritoneal surface malignancies. Ann Surg 2012; 255(2): 348-56.

[13] Yan TD, Links M, Fransi S, et al. Learning curve for cytoreductive surgery and perioperative intraperitoneal chemotherapy for peritoneal surface malignancy--a journey to becoming a Nationally Funded Peritonectomy Center. Ann Surg Oncol 2007; 14(8): 227080 .

[14] Kusamura S, Baratti D, Deraco M, et al. Learning curve for cytoreductive surgery and hyperthermic intraperitoneal chemotherapy in peritoneal surface malignancies: Analysis of two centres. J Surg Oncol 2013; 107(4): 312-9.

[15] Mohamed F, Moran BJ. Morbidity and mortality with cytoreductive surgery and intraperitoneal chemotherapy: the importance of a learning curve. Cancer J 2009; 15(3): 196-9.

[16] Jayne DG, Fook S, Loi C, Seow-Choen F. Peritoneal carcinomatosis from colorectal cancer. Br J Surg 2002; 89(12): 1545-50.

[17] Lemmens VE, Klaver YL, Verwaal VJ, Rutten HJ, Coebergh JW, de Hingh IH. Predictors and survival of synchronous peritoneal carcinomatosis of colorectal origin: a population-based study. Int $\mathrm{J}$ Cancer 2011; 128(11): 2717-25.

[18] Segelman J, Granath F, Holm T, Machado M, Mahteme H, Martling A. Incidence, prevalence and risk factors for peritoneal carcinomatosis from colorectal cancer. Br J Surg 2012; 99(5): 699705 .

[19] Gunderson LL, Sosin H, Levitt S. Extrapelvic colon-areas of failure in a reoperation series: implications for adjuvant therapy. Int $\mathrm{J}$ Radiat Oncol Biol Phys 1985; 11(4): 731-41.

[20] Tong D, Russell AH, Dawson LE, Wisbeck W. Second laparotomy for proximal colon cancer. Sites of recurrence and implications for adjuvant therapy. Am J Surg 1983; 145(3): 382-6.

[21] Cavaliere F, Valle M, Garofalo A, et al. 120 peritoneal carcinomatoses from colorectal cancer treated with peritonectomy and intra-abdominal chemohyperthermia: a S.I.T.I.L.O. multicentric study. In Vivo 2006; 20: 747-50. 
[22] Verwaal VJ, van Ruth S, de Bree E, et al. Randomized trial of cytoreduction and hyperthermic intraperitoneal chemotherapy versus systemic chemotherapy and palliative surgery in patients with peritoneal carcinomatosis of colorectal cancer. J Clin Oncol 2003; 21: 3737-43.

[23] Verwaal VJ, Bruin S, Boot H, van Slooten G, van Tinteren H. 8-year follow-up of randomized trial: cytoreduction and hyperthermic intraperitoneal chemotherapy versus systemic chemotherapy in patients with peritoneal carcinomatosis of colorectal cancer. Ann Surg Oncol 2008; 15: 2426-32.

[24] Yan TD, Cao CQ, Munkholm-Larsen S. A pharmacological review on intraperitoneal chemotherapy for peritoneal malignancy. World J Gastrointestinal Oncol 2010; 2(2): 109-16.
[25] Stephens AD, Alderman R, Sugarbaker PH, et al. Morbidity and mortality analysis of 200 treatments with cytoreductive surgery and hyperthermic intraoperative intraperitoneal chemotherapy using the coliseum technique. Ann Surg Oncol 1999; 6: 790-6.

[26] Glehen O, Osinsky D, Gilly FN, et al. Intraperitoneal chemohyperthermia usind a closed abdominal procedure and cytoreductive surgery for the treatment of peritonel carcinomatosis: morbidity and mortality analysis of 216 consecutive procedures. ANN Surg Oncol 2003; 10: 863-9.

[27] Kanellos D, Pramateftakis MG, Lazaridis H. The effects of the intraperitoneal administration of oxaliplatin and 5-FU on the healing of colonic anastomoses: an experimental study. Tech Coloproctol 2011.

(C) Kirby et al.; Licensee Bentham Open.

This is an open access article licensed under the terms of the Creative Commons Attribution Non-Commercial License (http://creativecommons.org/licenses/by-nc/3.0/) which permits unrestricted, non-commercial use, distribution and reproduction in any medium, provided the work is properly cited. 